\title{
Spatial knowledge during skilled action sequencing: Hierarchical versus nonhierarchical representations
}

\author{
Lawrence P. Behmer Jr. ${ }^{1}$ Matthew J. C. Crump ${ }^{1}$
}

Published online: 31 July 2017

(C) The Psychonomic Society, Inc. 2017

\begin{abstract}
Typists can type 4 to 5 keystrokes per second at around $95 \%$ accuracy, yet they appear to have poor declarative knowledge of key locations. Logan and Crump (2011, Psychology of Learning and Motivation, Vol. 54, pp. 1-27) accounted for this paradox by proposing that typing is hierarchically organized into two loops, with an outer loop that transforms sentences into words and passes each word, one at a time, to an inner loop that transforms each word into its constituent keystrokes; however, the nature of the inner loop's spatial knowledge is not well understood. Key locations may be learned through the experiences of locating and traversing between keys. In daily life, people tend to type structured language, and, as a consequence, certain keys and key-to-key transitions are experienced more frequently than others. Here, we asked whether or not this knowledge is structured hierarchically. For example, knowledge of key locations may be nested within representations of words, or the inner loop may rely on knowledge that is independent from higher level structures. To test this, we had people type English, English-like, and random strings during normal, partially occluded, and occluded typing. In both partially occluded and occluded typing, error rates were higher while typing random strings compared to English and English-like strings, whereas there was no difference in error rates between English and English-like strings. This suggests that typists' spatial knowledge of the keyboard is not driven by
\end{abstract}

Lawrence P. Behmer, Jr.

LBehmer@brooklyn.cuny.edu

Matthew J. C. Crump

mcrump@brooklyn.cuny.edu

1 Department of Psychology, Brooklyn College of the City University of New York, 2900 Bedford Avenue, Brooklyn, NY 11210, USA hierarchical word-level representations, but instead is likely driven by a collection of individual processes, such as knowledge of the sequential structure of language acquired by typing more frequently occurring letters.

Keywords Motor control $\cdot$ Spatial cognition

Spatial cognition is fundamental for goal-directed behavior across spatial scales, from navigating city streets to locating keys on a computer keyboard. To date, research has focused less on how people navigate in microenvironments, such as typing on a keyboard, and has instead focused more on spatial cognition in macro-environments, such as finding one's way from one point in town to another, or judging the distances and directions on a physical or mental map (Hintzman, O’Dell, \& Arndt, 1981; Ishikawa \& Montello, 2006; Maguire, Frackowiak, \& Frith, 1997; McNamara, 1986; McNamara, Ratcliff, \& McKoon, 1984; Siegel \& White, 1975; Thorndyke \& Hayes-Roth, 1982; Tversky, 1993, 2000). As a consequence, whether or not principles of spatial cognition in macro-environments apply across spatial scales to microenvironments, such as typing on a QWERTY keyboard, is not well known.

Theories of spatial cognition describe knowledge of spatial relations in terms of the form and function of underlying representations, the structure of how representations are coded with respect to one another, and whether the content of spatial information is embedded in representations or computed by processes operating on representations (McNamara, 1986). We use the first three concepts to guide our questions about how people acquire and use spatial knowledge about the keyboard during typing.

Spatial knowledge could be represented as analog forms such as mental images or memories of environments (Kosslyn, 1975; Kosslyn \& Pomerantz, 1977), in 
propositional forms such as using language to describe object positions (Pylyshyn, 1973), or both (Kosslyn \& Shwartz, 1977). Indeed, there is wide agreement that cognitive maps are not "map-like" and are better described as cognitive collages (Tversky, 1993, 2000) that combine multiple representational forms. So, typists may not have a high-fidelity internal map of the keyboard but instead could rely on multiple forms of spatial knowledge to guide their fingers during typing.

Different forms of representation provide different functions. If typists have different forms of spatial knowledge about the keyboard, they may use them in an ad hoc fashion. For example, an analog mental image of the keyboard would be useful for judging distances and angles between keys, and propositional knowledge that a keyboard has a QWERTY layout would be useful for recalling which letter is placed directly to the right of $Q$.

Spatial knowledge is acquired and developed with experience by processes that structure how new and old spatial knowledge is coded with respect to one another. Spatial knowledge about macro-environments can develop in a scaffolded manner, with initial learning about landmarks enabling learning about individual routes between landmarks, and learning about multiple routes enabling integrated highlevel survey or map-like knowledge about an environment (Siegel \& White, 1975). However, spatial knowledge does not necessarily progress through the above stages, and people can rely on minimally necessary spatial knowledge to accomplish spatial tasks without developing higher level integrated forms of spatial knowledge (Byrne, 1982). Typists may achieve the ability to navigate the keyboard perfectly well without forming a map-like representation of the keyboard, and instead rely on more local route-like knowledge for moving fingers from key to key.

A central question about processes that structure relations between spatial knowledge is whether relations are represented hierarchically (McNamara, 1986; Shelton \& McNamara, 2001; Stevens \& Coupe, 1978). Hierarchical representations group details of spatial codes into individual nested objects. For example, a New Yorker might represent New York City, the five boroughs, neighborhoods, and specific streets, routes, and buildings using connected but separate spatial codes. An open question is whether and how people integrate new spatial codes with existing nested representations. For example, Wang and Brockmole (2003) showed that people exposed to a new spatial environment (an experimenter's lab) within a familiar environment (university campus) can create new spatial representations that are not necessarily integrated with existing spatial knowledge. Similarly, in the present work we ask whether typists' knowledge of key locations on a QWERTY keyboard is coded hierarchically, by higher level word units that group together routes between key locations. We first review prior work examining the quality of typists' spatial knowledge of the QWERTY keyboard.

\section{Spatial knowledge of the QWERTY keyboard}

Experts can type four to six letters per second and maintain high (94\%) accuracy. On the surface, this suggests typists have high-fidelity spatial knowledge about key locations that enables speeded finger movements to individual keys. However, several findings suggest that this knowledge is not represented by an internal analog of the keyboard. First, typists have poor declarative knowledge of key locations on a keyboard. For example, Snyder, Ashitaka, Shimada, Ulrich, and Logan (2014) showed that typists could identify the correct key locations on a blank QWERTY keyboard only 57\% of the time in a pen-and-paper task. Accuracy increased to $79 \%$ when participants were cued to identify a specific key location but were still significantly worse than during normal typing (94\%). Second, typists perform poorly at estimating the distances and angle between keys (Liu, Crump, \& Logan, 2010). Typists apparently know where the keys are when they are typing, but not when they are explicitly asked to report on key locations. Finally, error rates during typing dramatically increase as kinematic and tactile cues from the keyboard are removed (Crump \& Logan, 2010). If typists had an internal analog map of the keyboard, they should have been able to explicit identify letter locations, judge angles and distances between keys, and move fingers to appropriate locations without external feedback.

\section{The task of typing is hierarchical}

The somewhat paradoxical finding that typists have excellent procedural knowledge and poor declarative knowledge of key locations can be explained by Logan and Crump's (2011) twoloop theory of typewriting. The theory proposes that fast and accurate typing is controlled by independently nested loops that divide the labor of typing. For example, the outer loop relies on language generation and comprehension to turn ideas into paragraphs, sentences, and words that are sent to the inner loop. The inner loop receives word-level instructions and serially orders keystroke responses to type each letter in a word. The division of labor explains the paradox, because the outer loop does not know the details of how the inner loop executes keystrokes. Similarly, knowledge of key locations is assumed to be housed by the inner loop.

This raises the question of how the inner loop knows where the keys are. If typists do not have a high-fidelity analog map of the keyboard, then what is the form of their spatial knowledge? We propose that knowledge of key locations are learned as individual landmarks or routes over the course experience with locating and traversing between particular keys. On this view, the inner loop does not have an integrated map of the keyboard but a parceled, memory-based collection of keystroke procedures (Logan, 1988; Rosenbaum et al., 1995). 
If the inner loop uses memory-based procedures for traversing between keys, then knowledge about individual key locations should depend on the frequency with which individual keys are typed. Indeed, prior work has established that interkeystroke intervals are shorter for more frequent than less frequent letters, bigrams, and trigrams (Behmer \& Crump, 2017b). This suggests that procedures for generating individual keystrokes are tuned by a process sensitive to the frequency of specific keystrokes. We are interested here in determining whether or not spatial knowledge about key locations and individual key transitions are structured in a hierarchical manner. For example, spatial knowledge about individual key locations may be cued by higher level structure at different $n$ gram levels or at the word level. Alternatively, the inner loop could form knowledge about key locations and transitions that are independent from higher level structure.

We tested these alternatives by comparing error rates for typing English words, English-like strings that approximated the bigram structure of English, and random strings. If spatial knowledge of key locations is hierarchically organized at the word level, then we expect lower error rates for English words compared to English-like and random strings. If spatial knowledge of key locations is hierarchically organized at the bigram level, then we expect lower error rates for English words and English-like strings compared to random strings, because words and word-like strings contain more frequently occurring and potentially well-learned keystroke transitions. If spatial codes are not hierarchically tied to higher level units, then we expect no differences in error rates at the letter level between string type conditions. For example, spatial knowledge of individual key locations could depend on key-specific practice such that key location knowledge is better for more than less frequently occurring letters. The main results sections of Experiments 1 and 2 address the evidence for hierarchical coding at the word and bigram level, and the final combined experiment analysis section addresses evidence for nonhierarchical coding at the letter level.

\section{Experiment 1}

Prior work shows that typists are faster when typing normal words compared to random strings and that error rates tend to be stable across string types (Gentner, Larochelle, \& Grudin, 1988; Shaffer \& Hardwick, 1968). However, differences in error rates may be obscured when typists can freely view the keyboard, because they may switch from relying on innerloop spatial knowledge to using visual cues when key locations are uncertain. Snyder, Logan, and Yamaguchi (2015) manipulated typists' access to visual cues by occluding the keyboard and withholding feedback from the monitor. They found that typists were slower and committed more errors when the keyboard and hands were occluded compared to normal typing. We adopted these occlusion manipulations to maximize reliance on inner-loop knowledge of key locations during typing.

In Experiment 1, typists copied normal English words, English-like nonwords that conformed to the bigram structure of the English language, and random strings while their hands and keyboard were visually occluded and typing output was withheld from the computer monitor. The central question of interest was whether error rates were lowest for words compared to other string types, which would indicate hierarchical spatial coding of key locations.

\section{Method}

Participants Fifty people participated in Experiment 1. Some participants had high error rates in the occluded condition. In order to analyze a common set of subjects on all dependent measures, we excluded participants with less than four correct RT responses in the occluded typing conditions (Van Selst \& Jolicouer, 1994). As a consequence, 12 participants were removed from the final analysis. This left a total of 38 participants (five males) in the final analysis. Participants' mean age was $20(S D=3.4)$ They typed an average of 64 words per minute $(S D=14)$, reported having been typing for 12 years $(S D=3.3)$, and started typing at 9 years of age $(S D=3.0)$. Eighteen participants reported that they had received some type of formal typing instruction, either during $\mathrm{K}-12$ or from a computer-based tutorial (mean training time $=25$ weeks, $S D$ $=22$ ). Fourteen participants self-reported as touch typists, and 11 self-reported as "hunt and peck" (nine other; four no response). All participants reported having everyday access to a computer. They reported using a computer an average of 4.5 hours a day $(S D=3.0)$ and spent an average of $41 \%$ of their time on a computer typing endogenously generated text $(S D=$ $33 \%)$ versus $14 \%$ copy typing $(S D=18 \%)$.

Stimuli All the stimuli were five-letter words. The English words were compiled from the MRC Psycholinguistic Database (Wilson, 1987) with a frequency range of 100 to 500. The English-like words were generated according to bigram probabilities from a large corpus of text that we compiled for a previous experiment by counting the occurrences of single letters, bigrams, and trigrams from more than 3,000 freely available e-books found on Project Gutenberg (Behmer \& Crump, 2016a). This resulted in generating fiveletter strings that, while not actual words, were generated based on the bigram frequency from our Gutenberg corpus. The random words were constructed by randomly sampling each letter in the alphabet with replacement leading to an equal likelihood of occurrence for each letter. Our stimuli controlled for physical constraints. There was an even distribution between single and two-handed bigrams (see Table 1). Additionally, we calculated the correlations between the 
Table 1 Distribution of unimanual and bimanual bigrams across block and string type in Experiment 1

\begin{tabular}{llll}
\hline Condition & & & \\
\hline Block & String type & Bigram distribution & $N$ \\
Normal & English & One hand & 147 \\
Normal & English & Two hand & 153 \\
Normal & English-like & One hand & 142 \\
Normal & English-like & Two hand & 157 \\
Normal & Random & One hand & 166 \\
Normal & Random & Two hand & 134 \\
Occluded & English & One hand & 171 \\
Occluded & English & Two hand & 129 \\
Occluded & English-like & One hand & 155 \\
Occluded & English-like & Two hand & 145 \\
Occluded & Random & One hand & 159 \\
Occluded & Random & Two hand & 141 \\
\hline
\end{tabular}

single-letter and bigram frequency of our stimuli with the single-letter and bigram frequency from our Gutenberg corpus. English (letters: $r=.87$; bigrams: $r=.64$ ) and Englishlike (letters: $r=.92$; bigrams: $r=.86$ ) strings were strongly correlated with the Gutenberg single-letter bigram frequency distributions; however, random strings (letters: $r=.04$; bigrams: $r=.03$ ) were not well correlated.

Design and procedure All procedures were conducted with the approval of the Brooklyn College Institutional Review Board. After reading and signing an informed consent, participants were brought into the experiment room to participate in the study. Up to four participants were run during each session, with each participant seated comfortably in a private cubicle under normal florescent lighting, within 55-60 cm of a Mac computer and standard IBM-clone QWERTY USB keyboard (see Fig. 1, left panel). During occluded typing, a cardboard box that was $42 \mathrm{~cm}$ in length $\times 31 \mathrm{~cm}$ wide $\times 21$ centimeters high covered the keyboard (see Fig. 1, right panel). The box had a $10-\mathrm{cm}$ tall opening in the front that ran the entire length of the box so that participants could comfortably fit their hands inside to type but were unable to see either their hands or the keyboard. During normal typing, the box was removed. Prior to the start of the experiment, participants practiced typing a short paragraph while the keyboard and their hands were occluded beneath the box. If a participant struggled during the occluded practice task, the box was temporarily removed and they were allowed to type a few sentences while being able to see their hands and the keyboard. When they were ready, the box was then put back in place and the participant continued practicing. If participants needed more practice, they were allowed to type the paragraph twice. During the actual occluded trials, the box was not removed until the block was completed.

The experiment was programmed using LiveCode 6.6.2. Prior to each trial, participants were instructed to rest their hands on the home row of the keyboard. Each trial (see Fig. 2) began with a fixation cross that appeared in the center of the screen for $500 \mathrm{~ms}$, followed by a $500 \mathrm{~ms}$ blank screen ISI. This was followed by the to-be-typed stimulus that remained on the screen for the duration of the trial. All stimuli were presented in the center of the screen in a 36 point Times New Roman font. The color of the text was black and presented against a gray background. Participants were instructed to respond to the word as quickly and accurately as possible. Participants did not receive correct/incorrect feedback. When participants typed a letter, regardless of whether or not the keystroke was correct or incorrect, the background of the letter changed from gray to green. After five keypresses, the next trial automatically began. During the task, the backspace key was disabled. There was a 2,000 ms pause between trials, so that participants could move their hands back to the home row of the keyboard. The three different types of word strings were presented in random order in each block. In total, participants typed 225 unique words in each block. Since we were concerned that typists might perform better during occluded typing after engaging in normal typing, blocks were not counterbalanced. Participants always performed the occluded typing block first, followed by the normal typing block. Mandatory 30 -s breaks occurred after 75 and 150 trials. After the completion of the experimental procedure, participants completed a survey that collected their basic demographic information and typing experience.

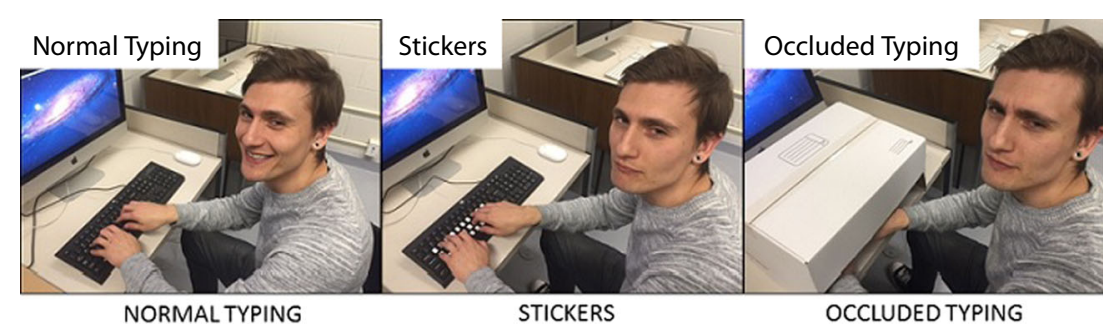

Fig. 1 Block manipulations. During the normal typing blocks (left panel) participants could see their hands and the keyboard while typing. In the stickers block (middle panel) participants could see their hands and the keyboard, but the individual keys were occluded with stickers. During the occluded block (right panel) participants' hands and the keyboard were occluded beneath a box 


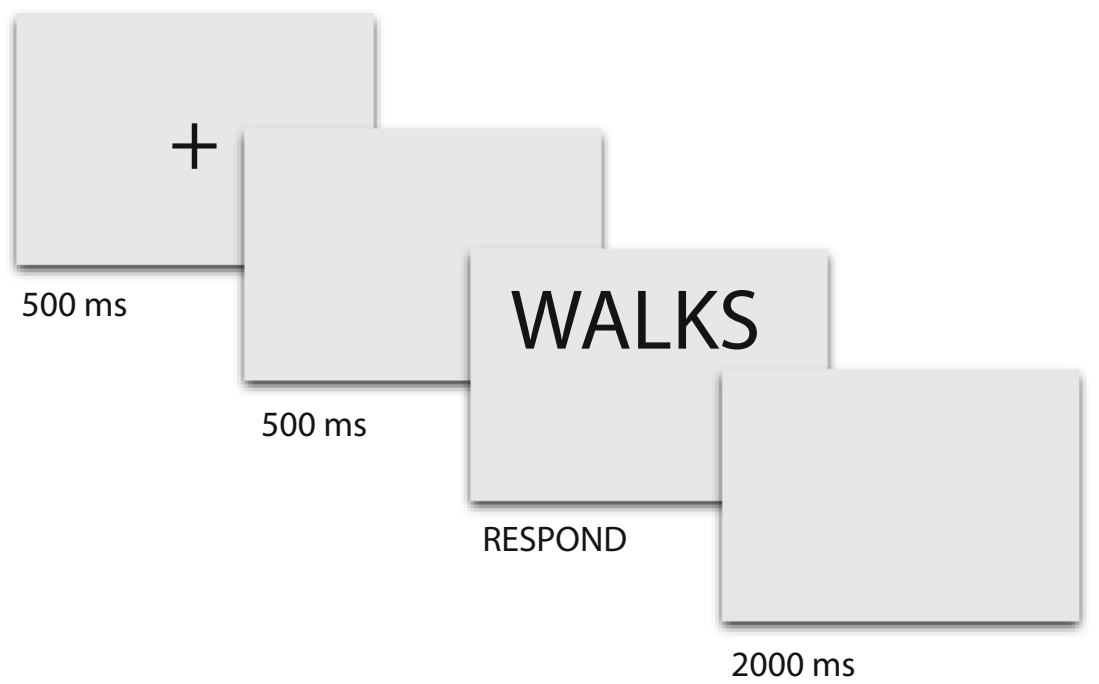

Fig. 2 Trial sequence. Each trial began with a fixation cross for $500 \mathrm{~ms}$, followed by a brief ISI ( $500 \mathrm{~ms}$.), and then a five-letter word. Participants were instructed to type the word as quickly and accurately as soon as it

\section{Results}

We collected error rates, reaction times (RTs; the time in milliseconds to type the first letter of the word), and interkeystroke intervals (IKSIs; the difference in time in ms between the current and previous keypress) in each condition. Correct RTs and IKSIs were submitted to an outlier elimination procedure (nonrecursive; Van Selst \& Jolicoeur, 1994) that removed an average of $3 \%$ of observations. For each subject, means from each condition were submitted to a 3 (string type: English, English-like, random string) $\times 2$ (keyboard occlusion: normal vs. occluded) repeated-measures ANOVA. Analyses for RTs and IKSIs were restricted to correct responses. Planned comparisons were performed using paired $t$ tests. Planned comparisons for a String Type $\times$ Keyboard Occlusion interaction were Bonferroni corrected to $p<.006$. Means and standard deviations for all measures in Experiment 1 can be found in Table 2.

Error rates There was a main effect of keyboard occlusion, $F(1,37)=88.38, M S E=0.094, p<.001, \eta_{\mathrm{p}}{ }^{2}=0.70 ;$ string

Table 2 Experiment 1 means and standard errors (in parentheses)

\begin{tabular}{llllll}
\hline \multicolumn{1}{l}{ Condition } & \multicolumn{3}{l}{ Measure } & & \\
\cline { 1 - 2 } \cline { 5 - 6 } Block & String type & & RT(ms) & IKSI(ms/keystroke) & ER (\%) \\
\hline Normal & English & & $779(20.4)$ & $171(6.4)$ & $4(0.01)$ \\
Normal & English-like & $869(24.6)$ & $240(11.4)$ & $4(0.01)$ \\
Normal & Random & $1,, 037(29.3)$ & $404(17.1)$ & $5(0.01)$ \\
Occluded & English & $867(33.6)$ & $259(17.1)$ & $41(0.04)$ \\
Occluded & English-like & $952(36.5)$ & $307(17.7)$ & $41(0.04)$ \\
Occluded & Random & $1,068(44.7)$ & $469(24.3)$ & $46(0.01)$ \\
\hline
\end{tabular}

was presented. Each trial was followed by a brief ISI. In Experiment 1, the posttrial ISI was 2,000 ms. In Experiment 2, it was 5,000 ms

type, $F(2,74)=23.03, M S E=0.0010, p<.001, \eta_{\mathrm{p}}{ }^{2}=0.38$; and a Keyboard Occlusion $\times$ String Type interaction, $F(2,74)=$ $6.87, M S E=0.0012, p=.003, \eta_{\mathrm{p}}{ }^{2}=0.16$. Figure 3 (far left panel) shows the mean error rates for Keyboard Occlusion $\times$ String Type. During occluded typing, error rates were lower for English, $t(37)=-4.70, p<.001$, and English-like strings, $t(37)=4.55, p<.001$, compared to random strings. There was no difference in error rates between English and English-like strings during occluded typing, $t(37)=-0.25, p=.80$. During normal typing, there was no difference between English and English-like, $t(37)=-1.27, p=.21$, or English-like and random strings, $t(37)=1.84, p=.07$. Although mean error rates were numerically higher for random than English strings, they were not statistically different according to .006 threshold for multiple comparisons, $t(37)=-2.90, p=.006$, Additionally, error rates were higher during occluded compared to normal typing when typing English, $t(37)=-9.25, p<.001$; Englishlike, $t(37)=-8.94, p<0.001$; and random strings, $t(37)=$ $-9.67, p<.001$.

Reaction times There was a main effect for keyboard occlusion, $F(1,37)=9.64, M S E=26863, p=.004, \eta_{\mathrm{p}}{ }^{2}=0.21$, and string type, $F(2,74)=163.03, M S E=6223, p<.001, \eta_{\mathrm{p}}{ }^{2}=$ 0.82 . There was also a Keyboard Occlusion $\times$ String Type interaction, $F(2,76)=5.07, M S E=3778, p=.09, \eta_{\mathrm{p}}{ }^{2}=$ 0.12 . Figure 3 (middle panel) shows the mean RTs for Keyboard Occlusion $\times$ String Type. RTs were faster during occluded typing for English compared to English-like, $t(37)=$ $-5.41, p<.001$, and random strings, $t(37)=-8.56, p<.001$. RTs were also faster for English-like compared to random strings, $t(37)=6.75, p<.001$. Likewise, RTs were faster during normal typing for English compared to English-like, $t(37)=-10.61, p<.001$, and random strings, $t(37)=-15.78, p$ 

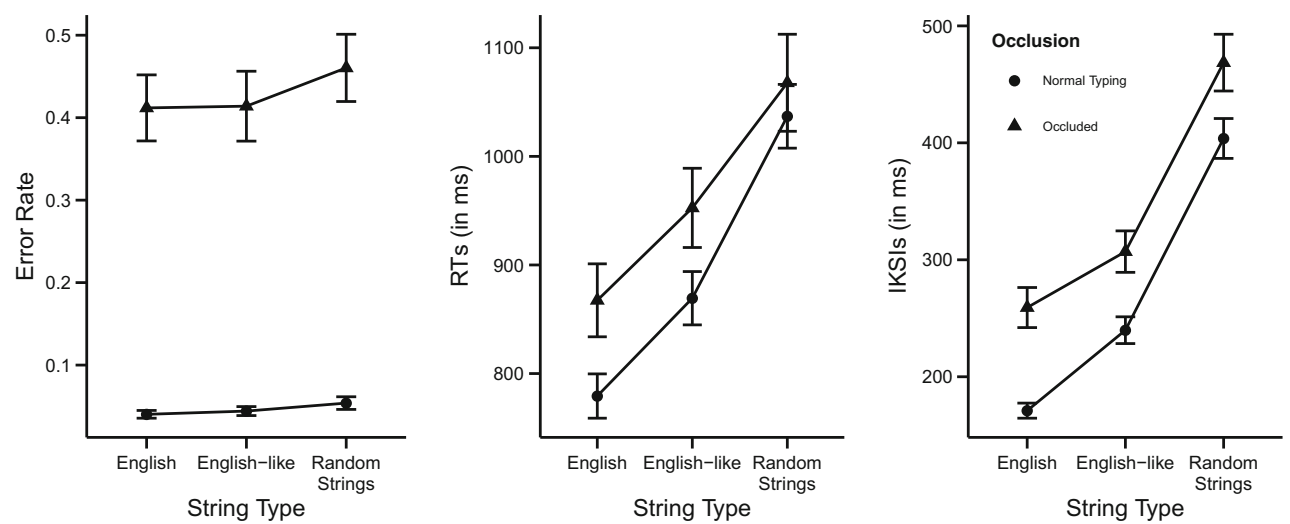

Fig. 3 Mean error rates (far left panel), RTs (middle panel), and IKSIs (far right panel) for Experiment 1 with standard error bars

$<.001$. RTs were also faster for English-like compared to random strings, $t(37)=13.49, p<.001$. Additionally, RTs were faster during normal compared to occluded typing when typing English, $t(37)=-4.17, p<.001$, and English-like strings, $t(37)=-4.08, p<.001$; however, there was no difference between normal and occluded typing when typing random strings, $t(37)=-1.00, p=.32$.

IKSIs There was a main effect for keyboard occlusion, $F(1$, $37)=38.46, M S E=7981, p<.001, \eta_{\mathrm{p}}{ }^{2}=0.51$, string type, $F(2,74)=292.25, M S E=3,413, p<.001, \eta_{\mathrm{p}}{ }^{2}=0.89$; however, there was not a Keyboard Occlusion $\times$ String Type interaction, $F(2,74)=2.87, M S E=1092, p=.07$. Figure $3($ far right panel) shows the mean IKSIs for Keyboard Occlusion $\times$ String Type. IKSIs were faster during normal compared to occluded typing, $t(37)=6.19, p<.001$. IKSIs were also faster when typing English compared to English-like, $t(37)=$ $-10.487, p<.001$, and random strings, $t(37)=-18.25, p<$ .001 , as well as while typing English-like compared to random strings, $t(37)=19.28, p<.001$.

\section{Discussion}

We were interested in investigating whether the nature of the inner-loop's knowledge of the spatial layout of the keyboard was hierarchical. We tested this by having participants type English words, English-like words constructed from the frequency distributions of bigrams in the English language, and random letter strings during normal typing conditions and when the keyboard and hands were occluded beneath a box. We observed that during occluded typing, error rates did not differ when participants typed English and English-like strings, and both were significantly lower compared to error rates when typing random strings. This suggests that the spatial codes between strings and their constituent key presses are not hierarchically linked to higher level units, such as words. If knowledge of key locations were driven by word-level hierarchies, then we would have expected error rates during occluded typing to be significantly lower for English words than English-like strings. Instead, the finding that error rates were higher for random strings than English words and English-like strings is consistent with hierarchical coding at the level of bigrams or key-to-key transitions, and consistent with nonhierarchical coding at the level of individual keys. These alternatives are addressed in the combined analysis of Experiment 2.

Last, typists were slower during occluded compared to normal typing. These general findings are consistent with prior work from Snyder et al. (2015) who also observed that RTs and IKSIs were slower when feedback from the monitor was withheld and typists' hands and keyboard were occluded from view, as well as Crump and Logan (2010), who observed that typing became progressively slower as kinematic feedback from the keyboard was manipulated.

\section{Experiment 2}

The purpose of Experiment 2 was to reproduce the basic findings of Experiment 1 and control for potential confounds that could have produced higher error rates for the random strings compared to the English and English-like strings. First, in the occluded condition of Experiment 1 typists were not given the opportunity to confirm that their fingers were starting in a home-row position, so they could have committed several proximity errors and inadvertently executed keypresses on adjacent keys that were within one to two key locations of the correct key. We performed an unreported analysis where we treated nearby incorrect responses as correct responses, and found that the although error rates were reduced overall, the pattern of higher error rates for random strings compared to English and English-like strings remained significant. To address the confound directly, in Experiment 2 we instructed participants to visually reorient their hands to the home row between trials during occluded typing. Additionally, we introduced a partial occlusion condition where participants could see the keyboard and their hands, but the identities of the letters on the keyboard were occluded with stickers. Last, 
following the analysis of Experiment 2 we performed two combined analyses of the occluded conditions from both experiments. The first examines the pattern of higher error rates for random strings varies by typing expertise. The second examines evidence for nonhierarchical coding at the letter level by determining whether error rates correlate with letter frequency from the natural English language, and whether error rate distributions at the letter level are similar across the string type conditions.

\section{Method}

Participants Thirty-seven people participated in Experiment 2. Five participants were removed because they failed to record at least four RTs for each string type during occluded typing, leaving a total of 32 participants (11 males) in the final analysis. Participants' mean age was $21(S D=3.3)$. They typed an average of 65 words per minute $(S D=15)$, reported having been typing for 12 years $(S D=3.9$ years $)$, and started typing at 9 years of age $(S D=3.9$ years). Ten participants reported that they had received some type of formal typing instruction, either during $\mathrm{K}-12$ or from a computer-based tutorial (mean training time $=23$ weeks, $S D=20$ ). Twelve participants self-reported as touch typists and eight selfreported as "hunt and peck" (five other; seven no response). Twenty-eight participants reported having everyday access to a computer (one no; three no response). Of those who reported having daily access to a computer, participants reported using a computer an average of 4 hours a day $(S D=3.2)$ and spent an average of $37 \%$ of their time on a computer typing endogenously generated text $(S D=26 \%)$ versus $13 \%$ copy typing $(S D=22 \%)$.

Design and procedure The design and procedure for Experiment 2 was identical to Experiment 1, with the following differences. In addition to normal and occluded typing, a third block was added in which typists could see their hands and the keyboard while typing, but the individual keys were occluded with stickers (see Fig. 1, middle panel). Adding this additional block in Experiment 2 meant that participants completed three blocks of 225 unique words in each block. In an attempt to increase accuracy during occluded typing, participants were instructed to visually confirm that their hands were on the home row between trials. As a consequence, the time between trials in Experiment 2 was increased from 2,000 ms to 5,000 ms. Additionally, blocks were counterbalanced using a Latin square. In Experiment 2, there was an even distribution between single and two-handed bigrams (see Table 3 ). Furthermore, English (letters: $r=.90$; bigrams: $r=.66$ ) and English-like (letters: $r=.96$; bigrams: $r=.88$ ) strings were strongly correlated with the Gutenberg single-letter and bigram distributions; however, random strings (letters: $r=$ -.10 ; bigrams: $r=.06$ ) were not well correlated.
Table 3 Distribution of unimanual and bimanual bigrams across block and string type in Experiment 2

Condition

\begin{tabular}{llll}
\hline Block & String Type & Bigram Distribution & $N$ \\
\hline Normal & English & One hand & 155 \\
Normal & English & Two hand & 145 \\
Normal & English-like & One hand & 153 \\
Normal & English-like & Two hand & 147 \\
Normal & Random & One hand & 148 \\
Normal & Random & Two hand & 152 \\
Stickers & English & One hand & 142 \\
Stickers & English & Two hand & 158 \\
Stickers & English-like & One hand & 141 \\
Stickers & English-like & Two hand & 159 \\
Stickers & Random & One hand & 150 \\
Stickers & Random & Two hand & 150 \\
Occluded & English & One hand & 142 \\
Occluded & English & Two hand & 158 \\
Occluded & English-like & One hand & 147 \\
Occluded & English-like & Two hand & 153 \\
Occluded & Random & One hand & 160 \\
Occluded & Random & Two hand & 140 \\
\hline
\end{tabular}

\section{Results}

We collected error rates, reaction times (RTs; the time in ms to type the first letter of the word), and interkeystroke intervals (IKSIs; the difference in time in milliseconds between the current and previous keypress) in each condition. Correct RTs and IKSIs were submitted to an outlier elimination procedure (nonrecursive; Van Selst \& Jolicoeur, 1994) that removed an average of $3 \%$ of observations. For each subject, means from each condition were submitted to a 3 (string type: English, English-like, random string) $\times 3$ (keyboard occlusion: normal, partially occluded, occluded) repeatedmeasures ANOVA. Analyses for RTs and IKSIs were restricted to correct responses. Planned comparisons were performed using paired $t$ tests. Planned comparisons for a String Type $\times$ Keyboard Occlusion interaction were Bonferroni corrected to $p<.003$. Means and standard deviations for all measures in Experiment 1 can be found in Table 4 .

Error rates There was a main effect for keyboard occlusion, $F(2,62)=43.70, M S E=0.080, p<.001, \eta_{\mathrm{p}}^{2}=0.59 ;$ string type, $F(2,62)=59.55, M S E=0.0039, p<.001, \eta_{\mathrm{p}}{ }^{2}=0.66$; and a Keyboard Occlusion $\times$ String Type interaction, $F(4$, $124)=12.80, M S E=0.002, p<.001, \eta_{\mathrm{p}}^{2}=0.29$. Figure 4 (far left panel) shows the mean error rates for Keyboard Occlusion $\times$ String Type. During occluded typing, error rates were lower for English, $t(31)=-6.09, p<.001$, and English- 
Table 4 Experiment 2 means and standard errors (in parentheses)

\begin{tabular}{|c|c|c|c|c|}
\hline \multicolumn{2}{|l|}{ Condition } & \multicolumn{3}{|l|}{ Measure } \\
\hline Block & String type & $\mathrm{RT}(\mathrm{ms})$ & IKSI(ms/keystroke) & $\mathrm{ER}(\%)$ \\
\hline Normal & English & $763(21.2)$ & $166(5.9)$ & $5(0.01)$ \\
\hline Normal & English-like & $863(27.4)$ & $217(9.0)$ & $7(0.01)$ \\
\hline Normal & Random & $1,024(35.7)$ & $388(16.0)$ & $10(0.02)$ \\
\hline Stickers & English & 789 (29.5) & $198(11.1)$ & $14(0.02)$ \\
\hline Stickers & English-like & $853(30.7)$ & $242(11.8)$ & $17(0.03)$ \\
\hline Stickers & Random & 994 (39.6) & $410(23.4)$ & $28(0.05)$ \\
\hline Occluded & English & $837(42.7)$ & $247(18.7)$ & $41(0.06)$ \\
\hline Occluded & English-like & $875(44.5)$ & $282(16.8)$ & $41(0.06)$ \\
\hline Occluded & Random & $1,002(46.5)$ & 455 (28.7) & $49(0.05)$ \\
\hline
\end{tabular}

like strings, $t(31)=6.83, p<.001$, compared to random strings. There was no difference in error rates between English and English-like strings during occluded typing, $t(31)=0.17, p=.87$. During partially occluded typing, error rates were lower for English, $t(31)=-7.61, p<.001$, and English-like strings, $t(31)=8.47, p<.001$, compared to random strings. The difference in error rates between English and English-like strings during occluded typing was not significant after correcting for multiple comparisons, $t(31)=-2.87, p$ $=.007$. During normal typing, there was no significant differences between English and English-like, $t(31)=-2.82, p=$ .008 , or English and random strings, $t(31)=-2.81, p=.009$, or English-like and random strings, $t(31)=2.45, p=.02$. Additionally, error rates were higher when typing English strings during occluded compared to partially occluded, $t(31)=-5.62, p<.001$, and normal typing, $t(31)=-6.67, p$ $<.001$, as well as during partially occluded compared to normal typing, $t(31)=-4.84, p<.001$. Likewise, error rates were higher when typing English-like strings during occluded compared to partially occluded, $t(31)=-5.68, p<.001$, and normal typing, $t(31)=-6.48, p<.001$, as well as during partially occluded compared to normal typing, $t(31)=-4.75, p<.001$. Finally, error rates were higher when typing random strings during occluded compared to partially occluded, $t(31)=$ $-6.31, p<.001$, and normal typing, $t(31)=-8.01, p<.001$, as well as during partially occluded compared to normal typing, $t(31)=-6.37, p<.001$.

Reaction times There was a main effect for string type, $F(2$, $62)=139.36, M S E=7962, p<.001, \eta_{\mathrm{p}}{ }^{2}=0.81$, and $\mathrm{a}$ Keyboard Occlusion $\times$ String Type interaction, $F(4,124)=$ $3.34, M S E=5622, p=.03, \eta_{\mathrm{p}}{ }^{2}=0.10$. There was no main effect for keyboard occlusion, $F(2,62)=0.62, M S E=29711$, $p=.51$. Figure 4 (middle panel) shows the mean RTs for Keyboard Occlusion $\times$ String Type. During occluded typing, RTs were faster for English, $t(31)=-5.60, p<.001$, and English-like strings, $t(31)=5.80, p<.001$, compared to random strings. There was no difference in RTs between English and English-like strings during occluded typing, $t(31)=$ $-1.25, p=.22$. During partially occluded typing, RTs were faster for English, $t(31)=-11.98, p<.001$, and English-like strings, $t(31)=10.98, p<.001$, compared to random strings, as well as for English compared to English-like strings, $t(31)=$ $-7.26, p<.001$. During normal typing, RTs were faster for English, $t(31)=-12.15, p<.001$, and English-like strings, $t(31)=11.71, p<.001$, compared to random strings, as well as for English compared to English-like strings, $t(31)=-9.83, p$ $<.001$.

IKSIs There was a main effect for keyboard occlusion, $F(2$, $62)=23.38, M S E=5178, p<.001, \eta_{\mathrm{p}}{ }^{2}=0.43$, and string type, $F(2,62)=229.82, M S E=5331, p<.001, \eta_{\mathrm{p}}^{2}=0.88$. There was not a Keyboard Occlusion $\times$ String Type interaction, $F(4,124)=0.71, M S E=868, p=.54$. Figure 4 (far right panel) shows the mean IKSIs for Keyboard Occlusion $\times$ String Type. IKSIs were faster during normal compared to partially occluded, $t(31)=-24.90, p<.001$, and occluded typing, $t(31)=5.98, p<.001$, as well as during partially
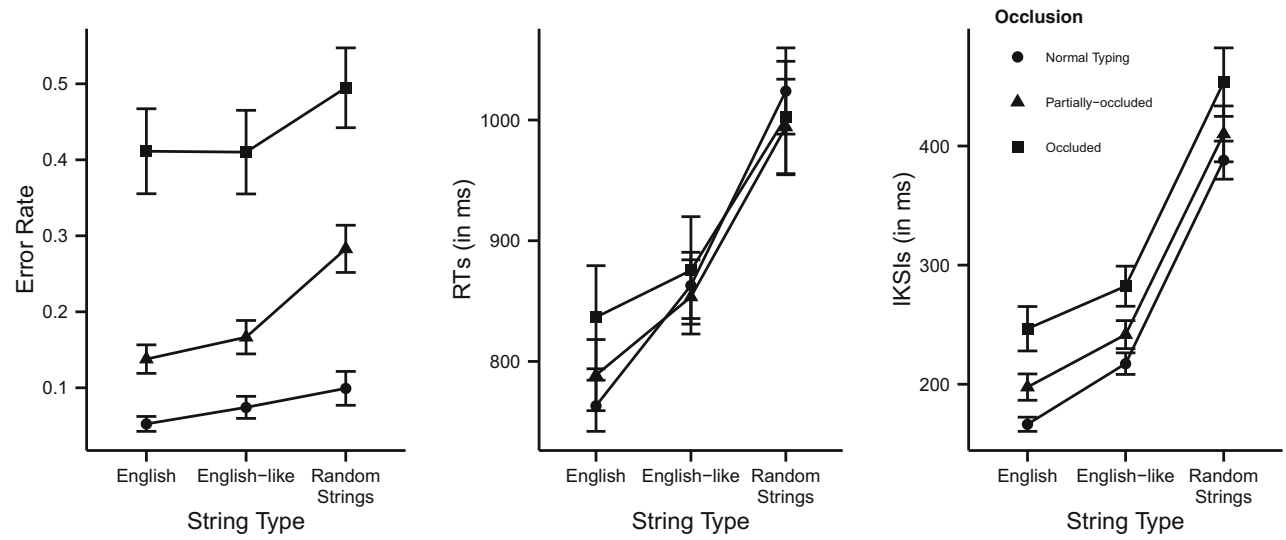

Fig. 4 Mean error rates (far left panel), RTs (middle panel), and IKSIs (far right panel) for Experiment 2 with standard error bars 
occluded compared to occluded typing, $t(31)=15.65, p<$ .001. Additionally, IKSIs were faster while typing English compared to English-like, $t(31)=-12.25, p<.001$, and random strings, $t(31)=-17.30, p<.001$, as well as for Englishlike compared to random string types, $t(31)=16.35, p<.001$.

Analysis of expertise effects Across two experiments we found that error rates are higher for random strings than English and English-like strings when the keyboard is occluded. This suggests, first, that spatial knowledge of key locations is not bound hierarchically to word-level representations, because we did not find any evidence of lower error rates for English compared to English-like strings. These findings stem from a group-level analysis, and the possibility remains that spatial knowledge of key locations depends on typing expertise. For example, reliance on hierarchical forms of spatial knowledge might develop with expertise, in which case experts rather than novices may show lower error rates for English compared to English-like strings. Alternatively, high-fidelity spatial knowledge of key locations may develop with expertise, in which case experts may be completely insensitive to the string-type manipulation showing no differences in error rates during occluding typing. Finally, expert typists may be more accurate compared to novice typists yet still show the same pattern of error rates observed in Experiment 1 and 2.

We used typing speed as a proxy for expertise and combined subjects from Experiments 1 and 2 . Specifically, each subject's normal typing speed was measured by their mean IKSI from the normal typing condition (i.e., English words, no occlusion). Then, subjects were grouped into fast and slow typists by a median split on typing speed. For each subject, mean error rates from each condition were submitted to a 2 (typing speed: fast, slow) $\times 3$ (string type: English, English-like, random) $\times 2$ (keyboard occlusion: normal vs. occluded) mixed-design ANOVA, with typing speed as the between-subjects variable, and string type and keyboard occlusion as withinsubjects variables. Mean error rates in each condition are shown in Fig. 5.

We found a main effect for typing speed, $F(1,68)=6.22$, $M S E=0.126, p=.03, \eta_{\mathrm{p}}{ }^{2}=0.06$; a main effect for keyboard occlusion, $F(1,68)=142.98, M S E=0.103, p<.001, \eta_{\mathrm{p}}{ }^{2}=$ 0.68 ; and a main effect for string type, $F(2,136)=46.28, M S E$ $=0.002, p<.001, \eta_{\mathrm{p}}{ }^{2}=0.40$. We also observed interactions for Typing Speed $\times$ Keyboard Occlusion, $F(1,68)=3.86$, $M S E=0.103, p=.05, \eta_{\mathrm{p}}{ }^{2}=0.05$, and Keyboard Occlusion $\times$ String Type, $F(2,136)=15.06, M S E=0.001, p<.001, \eta_{\mathrm{p}}{ }^{2}$ $=0.18$. There was no difference in error rates during normal typing between fast $(M=5 \%, S E=0.01)$ and slow typists $(M$ $=7 \%, S E=0.01), t(34)=0.92, p=.36$, nor was there a difference between fast $(M=37 \%, S E=0.03)$ and slow typists $(M=50 \%, S E=0.03)$ during occluded typing, $t(34)=1.90, p$
Occlusion Normal Typing Occluded

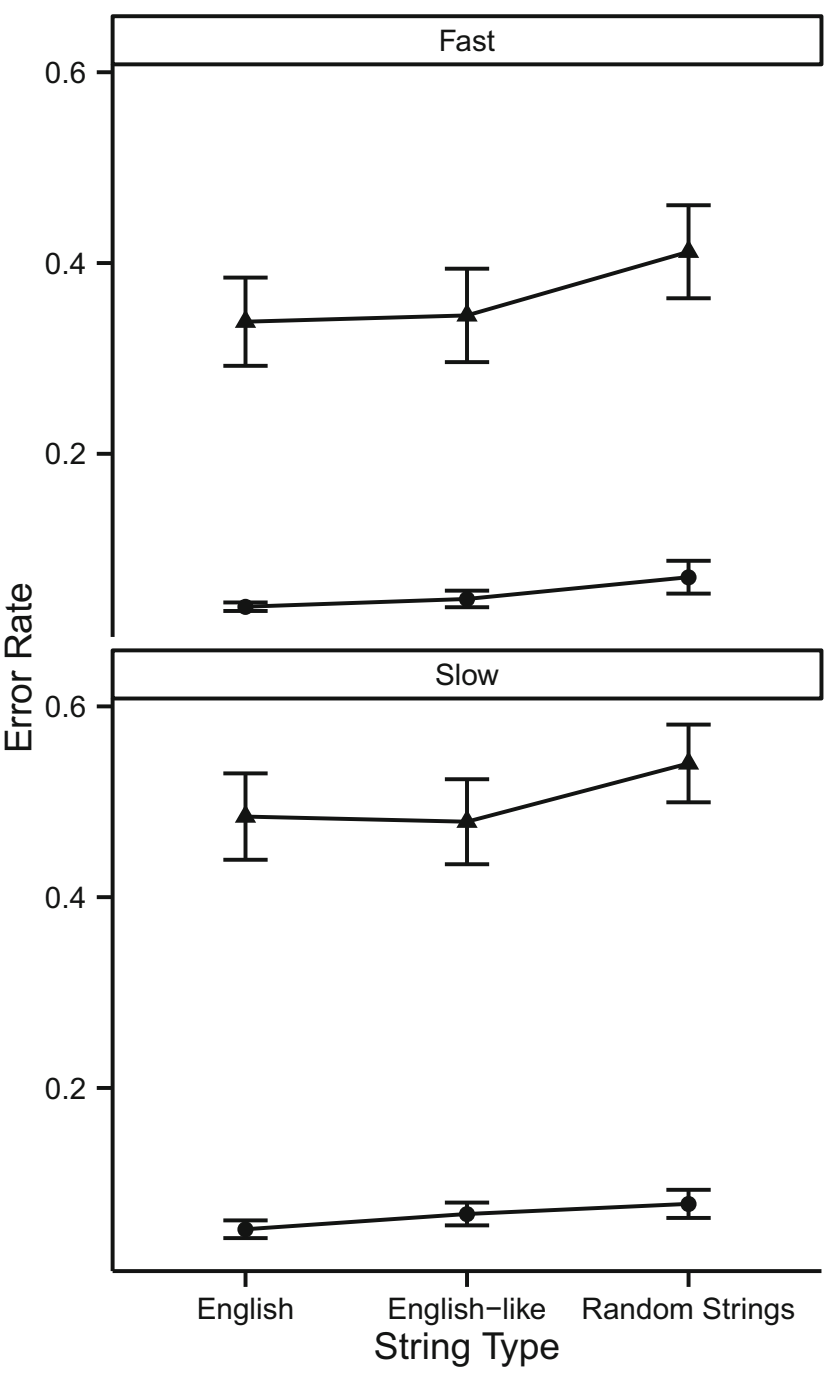

Fig. 5 Mean error rates for between-subjects expertise analysis, with standard error bars for the Keyboard Occlusion $\times$ Typing Speed

$=.07$. Fast, $t(34)=-10.27, p<.001$, and slow typists, $t(34)=$ $-6.79, p<.001$, had significantly higher error rates during occluded compared to normal typing. Importantly, the pattern of data for both fast and slow typists (see Fig. 5) looks almost identical to what was reported in Experiments 1 and 2. During occluded typing, error rates were lower for English $(M=41 \%$, $S E=0.03), t(69)=-7.50, p<.001$, and English-like strings $(M=41 \%, S E=0.03), t(69)=-7.80, p<.001$, compared to random strings $(M=47 \%, S E=0.03)$. There was no difference in error rates between English and English-like strings during occluded typing, $t(69)=-0.11, p=.91$. During normal typing, error rates were lower for English $(M=5 \%, S E=0.01)$ compared to English-like $(M=6 \%, S E=0.01), t(69)=-3.00$, $p=.004$, and random strings $(M=7 \%, S E=0.01), t(69)=$ $-3.50, p<.001$, as well as for English-like compared to random strings, $t(69)=-3.03, p=.003$. Additionally, error rates were higher during occluded compared to normal typing when 
typing English, $t(69)=-11.21, p<.001$, English-like, $t(69)=$ $-10.91, p<.001$, and random strings, $t(69)=-12.60, p<$ .001 .

Letter frequency and key-location knowledge In the introduction, we suggested that the procedures for generating individual keystrokes are tuned by a process sensitive to the frequency of specific keystrokes. Typists may have better spatial knowledge of the key locations for more frequently occurring letters because in natural language, some letters occur more frequently than other letters, and as a consequence are typed more often. If typists are more error prone for lower frequency letters, then our main finding that error rates are higher for random strings compared to English and English-like strings could be explained by the fact that random strings are more likely to contain lower frequency letters that would, in turn, increase error rates. We tested this possibility by determining whether letter-level error rates were correlated with letter likelihood.

Behmer and Crump (2017b) showed that typists' interkeystrokes intervals were negatively correlated with letter, bigram, and trigram frequencies (i.e., faster keystrokes for more than less frequent $n$-grams), but they did not determine whether error rates for specific letters depended on letter frequency. In their analysis, $n$-gram frequencies in the natural language were determined by counting letter, bigram, and trigrams from 3,000 randomly selected English language ebooks from the online digital repository Project Gutenberg. We used the letter frequencies from that analysis to determine whether error rates for individual letters in the fully occluded typing conditions from Experiment 1 and 2 were correlated with letter likelihood. In each string type condition, we computed the mean error rate for each letter collapsing across subjects and correlated the error rates with letter probabilities. The results displayed in Fig. 6 show that letter-specific error rates were negatively correlated with letter probability for all string type conditions (English: $r^{2}=-0.53, p<.001$; English- like: $r^{2}=-0.28, p<.007$; random string: $\left.r^{2}=-0.44, p<.001\right)$. Typists were more likely to make errors for letters that appear with lower than higher frequency in the natural language.

Letter-level error rates The major question of interest in this article was whether spatial knowledge of key locations is hierarchically organized by word-level units. If so, error rates for specific letters should be lower when they occur in strings with word-like versus non-word-like structure. If spatial knowledge of key locations is not hierarchically organized at the word level, then error rates for specific letters should be consistent across manipulations of string type. In the primary analyses of Experiment 1 and 2 we found that word-level error rates were higher for random strings than for English and English-like strings. However, as the above correlational analysis shows, it possible that the higher error rates for random strings was caused by subjects making more letter level errors for low-frequency letters, which are more common in the random string condition. To address this possibility more directly, we determined whether the pattern of error rates for individual letters was consistent across string types.

For each subject in Experiments 1 and 2, we calculated mean error rates from the occluded typing condition for each letter, separately for each string type condition. The error rates for five letters $(b, j, x, q, z)$ were undetermined for some subjects in some conditions because those letters did not occur in the presented strings. The mean error rates for the remaining 21 letters in each condition were submitted to a 3 (string type: English, English-like, and random) $\times 21$ (letter) repeatedmeasures ANOVA. Mean error rates for each letter in each condition are shown in Fig. 7.

There was a significant main effect of string type, $F(2,138)$ $=23.52, M S E=0.031, p<.001, \eta_{\mathrm{p}}{ }^{2}=.19$; a significant main effect of letter, $F(20,1380)=6.46, M S E=0.075, p<.001, \eta_{\mathrm{p}}{ }^{2}$ $=.06$; and a significant two-way interaction, $F(40,2760)=$ 2.27, MSE $=0.017, p<.001, \eta_{\mathrm{p}}{ }^{2}=.03$. Most important, was that mean error rates were highest in the random (.47),

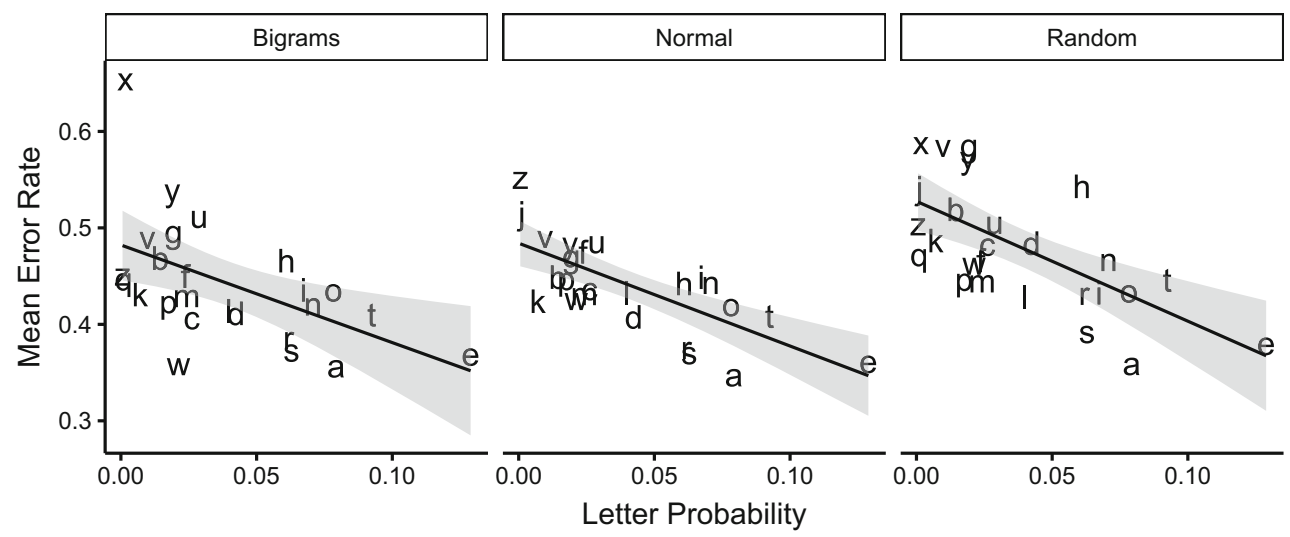

Fig. 6 Scatterplots showing the negative correlations between letter-level error rates ( $y$-axis) single-letter frequency distributions from the Gutenberg corpus ( $x$-axis). Error rates were collapsed across subjects from Experiments 1 and 2 and taken from the fully occluded condition 
Condition $-0-$ English $-\Delta \cdot$ English-like $-\square \cdot$ Random

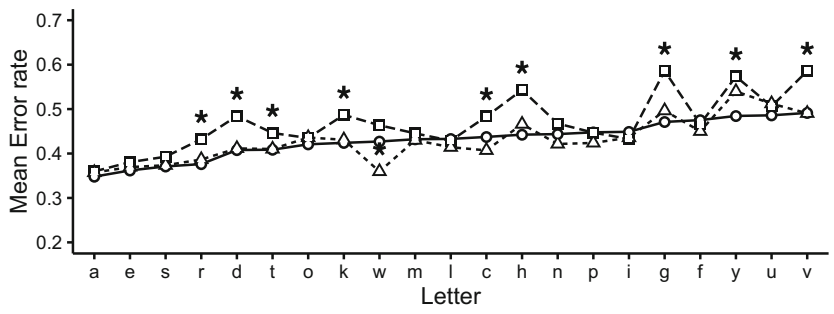

Fig. 7 Mean letter-level error rates by string type condition collapsed across subjects from Experiments 1 and 2. Letters are ordered from smallest to largest error rate for normal words, and asterisks indicate a significant difference compared to the normal error rate for the given letter

compared to the English (.43), and English-like (.43) conditions. As depicted in the figure, the analysis of simple effects showed that error rates for nine letters $(r, d, t, k, c, h, g, y, v)$ were significantly higher in the random string than English word condition. We also compared error rates between English and English-like string types and found only the error rate for w was significantly lower for English-like strings than English words. Data from all simple effect comparisons are presented in Table 5.

\section{Discussion}

We replicated our error rate findings from Experiment 1 after controlling for possible confounds associated with starting hand position during occluded typing. Furthermore, in Experiment 2, we introduced a partially occluded condition in which typists could see their hands and the keyboard, but the individual key locations were obscured by stickers. Although error rates improved during partially occluded compared to occluded typing, typists still showed lower error rates when typing English and English-like strings compared to random strings.

We did not replicate the main effect of keyboard occlusion that we observed in Experiment 1. This may have been the result of allowing participants to reorient their hand position to the home row between trials. As a consequence, participants may have been more confident of their initial response during occluded typing.

We also reported two analyses combining results across subjects in the occluded typing conditions from Experiment 1 and 2. The expertise analysis showed that both fast and slow typists showed the main pattern of higher error rates for random strings compared to English words and English-like

Table 5 Means and effects for letter-level analysis

\begin{tabular}{|c|c|c|c|c|c|c|c|}
\hline & \multirow{2}{*}{$\begin{array}{l}\text { English } \\
\operatorname{MER}(S E)\end{array}$} & \multirow{2}{*}{$\begin{array}{l}\text { English-like } \\
\text { MER (SE) }\end{array}$} & \multirow{2}{*}{$\begin{array}{l}\text { Random } \\
\text { MER (SE) }\end{array}$} & \multicolumn{2}{|c|}{ English vs. English-like } & \multicolumn{2}{|c|}{ English vs. Random } \\
\hline & & & & $F(M S E)$ & $P$ & $F(M S E)$ & $P$ \\
\hline $\mathrm{a}$ & $.47(.042)$ & $.48(.042)$ & $.48(.042)$ & $.59(.004)$ & .446 & $.54(.006)$ & .465 \\
\hline $\mathrm{c}$ & $.55(.04)$ & $.52(.042)$ & $.58(.037)$ & $2.07(.017)$ & .154 & $3.66(.014)$ & .059 \\
\hline $\mathrm{d}$ & $.51(.039)$ & $.52(.038)$ & $.58(.037)$ & $.23(.012)$ & .634 & $11.76(.017)$ & .001 \\
\hline $\mathrm{e}$ & $.48(.04)$ & $.49(.04)$ & $.49(.039)$ & $.46(.003)$ & .501 & $1.21(.004)$ & .275 \\
\hline$f$ & $.57(.038)$ & $.54(.04)$ & $.56(.038)$ & $1.33(.033)$ & .252 & $.16(.021)$ & .686 \\
\hline $\mathrm{g}$ & $.56(.037)$ & $.59(.038)$ & $.66(.034)$ & $1.71(.017)$ & .194 & $21.86(.018)$ & .000 \\
\hline $\mathrm{h}$ & $.55(.039)$ & $.56(.039)$ & $.62(.036)$ & $.84(.011)$ & .362 & $10.61(.022)$ & .002 \\
\hline $\mathrm{i}$ & $.55(.037)$ & $.54(.038)$ & $.53(.038)$ & $.74(.005)$ & .392 & $.84(.008)$ & .363 \\
\hline $\mathrm{k}$ & $.53(.039)$ & $.53(.041)$ & $.57(.036)$ &.$(.033)$ & .969 & $3.96(.022)$ & .049 \\
\hline 1 & $.54(.038)$ & $.52(.041)$ & $.53(.039)$ & $1.43(.012)$ & .235 & $.14(.01)$ & .708 \\
\hline $\mathrm{m}$ & $.53(.041)$ & $.53(.042)$ & $.55(.041)$ & $.01(.018)$ & .921 & $.93(.017)$ & .339 \\
\hline $\mathrm{n}$ & $.55(.04)$ & $.53(.04)$ & $.57(.039)$ & $2.07(.007)$ & .154 & $2.08(.009)$ & .153 \\
\hline o & $.52(.038)$ & $.54(.039)$ & $.54(.039)$ & $1.92(.007)$ & .169 & $.71(.01)$ & .403 \\
\hline $\mathrm{p}$ & $.55(.039)$ & $.53(.044)$ & $.54(.036)$ & $.44(.03)$ & .511 & $.58(.015)$ & .448 \\
\hline $\mathrm{r}$ & $.49(.038)$ & $.5(.039)$ & $.53(.038)$ & 1. (.005) & .321 & $5.99(.013)$ & .016 \\
\hline s & $.49(.041)$ & $.49(.04)$ & $.51(.04)$ & $.01(.006)$ & .917 & $2.15(.007)$ & .146 \\
\hline $\mathrm{t}$ & $.52(.04)$ & $.52(.038)$ & $.55(.037)$ & $.01(.005)$ & .936 & $4.75(.009)$ & .032 \\
\hline $\mathrm{u}$ & $.58(.039)$ & $.6(.038)$ & $.59(.037)$ & $.94(.014)$ & .336 & $.36(.011)$ & .551 \\
\hline $\mathrm{v}$ & $.59(.044)$ & $.58(.041)$ & $.66(.033)$ & $.05(.033)$ & .824 & $6.56(.036)$ & .012 \\
\hline $\mathrm{w}$ & $.54(.041)$ & $.48(.047)$ & $.56(.037)$ & $4.2(.031)$ & .044 & $.95(.024)$ & .333 \\
\hline y & $.57(.039)$ & $.62(.037)$ & $.65(.038)$ & $3.79(.024)$ & .055 & $14.42(.017)$ & .000 \\
\hline
\end{tabular}

MER = mean error rate; $S E=$ standard error of the mean 
strings. The letter-level analyses determined whether the higher error rates in the random string condition were due to differences in spatial knowledge at the letter level or at the level of key-to-key transitions. We first showed that letterlevel error rates negatively correlate with the frequency of letters in natural English, suggesting that typists knowledge of key locations is tied to the number of times they have typed each key. This finding could have explained the pattern of higher error rates for random strings compared to English and English-like conditions because random strings are more likely to contain more error-prone low-frequency letters. However, the second letter-level analysis ruled out this explanation.

The two important results from the second analysis were a main effect of string type and a String Type $\times$ Letter interaction. The main effect of string type showed that letters in the random string condition had higher error rates than letters in the English and English-like conditions. However, the interaction showed that only a subset of letters had higher error rates in the random than in the English and English-like conditions. The main effect suggests that some higher order linguistic structure (Pinet, Ziegler, \& Alario, 2016; Scaltritti, Arfé, Torrance, \& Peressotti, 2016) beyond the letter and bigram levels influenced typing accuracy. The presence of an interaction was unexpected, and we had no a priori predictions that the random string condition would systematically inflate the error rates for specific letters. We interpret this result with some caution. On the one hand, spatial knowledge of key locations may be nonhierarchical for some letters and partially hierarchical for others. On this view, future work is needed to determine what kind of hierarchical structure guides spatial knowledge for specific letters and not others, and how this influence changes with practice. On the other hand, the interaction could reflect low power to detect small differences in error rates at the letter level. For example, 17 of the 21 letters had numerically higher mean error rates in the random string than normal English conditions, but we could only detect statistically significant differences in nine of the letters. On this view, higher order linguistic structure may guide spatial knowledge for all letters, but the size of the effect at the letter level may be influenced by other factors including key position and letter and bigram frequency. Regardless, the most important conclusion was that error rates for specific letters were higher in the random than in the English and English-like conditions, showing that the higher error rates for the random strings in general were not entirely explained by letter frequency confounds.

\section{General discussion}

We set out to determine whether spatial knowledge of key locations has hierarchical or nonhierarchical structure. If knowledge of key locations is structured hierarchically, then typists' accuracy for typing individual keys should depend on whether a current letter is presented within an organized format, such as a word or bigram. If knowledge of key location is not structured hierarchically, then typists' accuracy for typing individual letters should not depend on the local bigram or larger word context in which they appear. Experiments 1 and 2 tested these ideas by examining whether error rates would vary as a function of the structure of string types, especially under conditions of occlusion forcing typists to rely on their prior spatial knowledge of the keyboard. In Experiment 2, we were able to replicate our findings from Experiment 1, showing that typists were more accurate for words that conformed to the structure of the English language compared to random strings. In Experiment 2, we introduced an intermediate condition in which typists could see their hands and the keyboard, but the individual keys were occluded with stickers. While error rates improved during this condition, compared to occluded typing, participants were still more accurate for English and English-like strings compared to random strings.

Over two experiments we found no evidence that error rates depended on word-level structure, as error rates for typing English words and English-like strings were equivalent. Instead, error rates were higher for random strings than for English words and English-like strings during both experiments. This finding was consistent with the idea that knowledge of key locations depends to some extent on bigram structure. Specifically, words and English-like words contain more high frequency key-to-key transitions than did random strings. At the same time, the higher error rates for random words is consistent with the idea that knowledge of key locations is nonhierarchical and depends on frequency of experience with typing specific letters that is independent from higher order sequential structure. The combined experiment letter-level error rate analyses showed some support for partly hierarchical and nonhierarchically organized knowledge of key locations. First, letter-level error rates did correlate with letter frequency from the natural language, showing that typists are more error prone for lower frequency letters. This finding could have accounted entirely for the higher error rates for random strings, as these strings would tend to have more lower frequency letters that would inflate the error rate compared to the other string type conditions. However, we also found that a small set of eight letters had higher error rates when subjects typed random strings compared to English words; whereas, the remaining letters showed equivalent error rates. This finding shows that knowledge of key locations is partly hierarchical (in that the accuracy of locating keys for a small set of letters depends on local bigram structure) and mainly nonhierarchical (because accuracy for most letters did not vary across the string type conditions).

An overarching question in spatial cognition research is the extent to which principles of spatial cognition apply across 
spatial scales. Pursuing this issue, we used skilled typing as a model task to study spatial knowledge of the keyboard microenvironment that supports accurate typing. Our results bolster support for the conclusion from prior work that typists do not have a high-fidelity, mental-image analog of the keyboard but instead rely on a collection of learned procedures for executing specific keystrokes.

Under normal typing conditions, experts can type very quickly and accurately. This implies that typists must have some form of high-fidelity knowledge of key locations. A high-quality mental-analog representation of the keyboard could in principle be used to for directing movements toward keys. However, use of a map-like representation should also generalize beyond typing to support recall of key identities and judgments of distances and angles between keys. However, prior work shows that typists perform poorly on those nontyping spatial tasks about key locations (Liu et al., 2010; Snyder et al., 2014). Additionally, if typists relied on a mental map-like representation, then error rates should not have increased under the present occlusion conditions, and error rates should not have varied as a function of string type or letter frequency.

The difference in error rates between occluded and normal typing suggests that typists can rely on several different forms of information that contribute to the fidelity of their spatial map of the keyboard. Typists were worst under full occlusion, suggesting that visual features from the keyboard and hands are important cues for locating keys. Typists were much better under partial occlusion when the identities of the letters were obscured by stickers, but still worse than normal typing. This finding shows that even when letters are not displayed on the keys, familiar visual cues about key, hand, and finger position are important cues for locating keys. It remains unclear whether all or some of these cues are used during normal typing. For example, typists may normally rely on key identity and location cues but switch to finger and hand position cues only when letter identities are hidden. We suspect in general that typists will use the best cues available to them under different typing conditions. This idea could explain why error rates were higher for the random strings only under conditions of occlusion, as typists may not rely strongly on letter frequency and letter context under normal conditions. At the same time, even under normal typing conditions, error rates were numerically higher in the random than in the English and Englishlike conditions but were not significant according to our conservative corrections for multiple comparisons. So, it remains possible that typists do rely on these cues during normal typing, but their influence is small and difficult to detect.

We argue that typists do not rely on an integrated high-level spatial representation of the keyboard but instead rely on a collection of individual procedures for executing specific keystrokes. We found that these procedures are largely nonhierarchical and appear to be tuned to the demands of executing specific keystrokes independent from neighboring keystrokes. In particular, letter-level error rates correlate with letter frequency, suggesting that the quality of keystroke procedures is related in a straightforward manner to keystroke-specific practice.

We did find that error rates for some letters were influenced by higher level structure at the word or bigram level, which indicates some variability in whether keystroke procedures are controlled in a hierarchical fashion. This finding raises further questions about why the surrounding letter context matters for typing particular letters but not others. One direction for future research is to examine changes in spatial knowledge over the course of learning to type on the keyboard. For example, it is unclear whether spatial knowledge of the keyboard becomes more hierarchical or more nonhierarchical with practice and expertise.

Author note This work was supported by a grant from NSF (\#1353360).

\section{References}

Behmer, L. P., Jr., \& Crump, M. J. C. (2017a). Crunching big data with finger tips: How typists tune their performance toward the statistics of natural language. In M. Jones (Ed.), Big data in cognitive science (pp. 1-28). London: Routledge.

Behmer, L. P., Jr., \& Crump, M. J. C. (2017b). The dynamic range of response set activation during action sequencing. Journal of Experimental Psychology: Human Perception and Performance, 43(3), 537-554.

Byrne, R. W. (1982). Geographical knowledge and orientation. In A. W. Ellis (Ed.), Normality and pathology in cognitive functions (pp. 239-264). London: Academic Press.

Crump, M. J. C., \& Logan, G. D. (2010). Warning: This keyboard will deconstruct-The role of the keyboard in skilled typewriting. Psychonomic Bulletin \& Review, 17, 394-399. doi:10.3758/PBR. 17.3.394

Gentner, D., Larochelle, S., \& Grudin, J. (1988). Lexical, sublexical, and peripheral effects in skilled typewriting. Cognitive Psychology, 20, 524-548.

Hintzman, D. L., O’Dell, C. S., \& Arndt, D. R. (1981). Orientation in cognitive maps. Cognitive Psychology, 13, 149-206. doi:10.1016/ 0010-0285(81)90007-4

Ishikawa, T., \& Montello, D. R. (2006). Spatial knowledge acquisition from direct experience in the environment: Individual differences in the development of metric knowledge and the integration of separately learned places. Cognitive Psychology, 52, 93-129.

Kosslyn, S. M. (1975). Information representation in visual images. Cognitive Psychology, 7, 341-370.

Kosslyn, S. M., \& Pomerantz, J. R. (1977). Imagery, propositions, and the form of internal representations. Cognitive Psychology, 9, 52-76.

Kosslyn, S. M., \& Shwartz, S. P. (1977). A simulation of visual imagery. Cognitive Science, 1, 265-295.

Liu, X., Crump, M. J. C., \& Logan, G. D. (2010). Do you know where your fingers have been? Explicit knowledge of the spatial layout of the keyboard in skilled typists. Memory \& Cognition, 38(4), 474484.

Logan, G. D. (1988). Toward an instance theory of automatization. Psychological Review, 95(4), 492-527. 
Logan, G. D., \& Crump, M. J. C. (2011). Hierarchical control of cognitive processes. In B. H. Ross (Ed.), Psychology of learning and motivation (Vol. 54, pp. 1-27). Amsterdam: Elsevier.

Maguire, E. A., Frackowiak, R. S., \& Frith, C. D. (1997). Recalling routes around London: Activation of the right hippocampus in taxi drivers. The Journal of Neuroscience, 17, 7103-7110.

McNamara, T. P. (1986). Mental representations of spatial relations. Cognitive Psychology, 18, 87-121. doi:10.1016/0010-0285(86) 90016-2

McNamara, T. P., Ratcliff, R., \& McKoon, G. (1984). The mental representation of knowledge acquired from maps. Journal of Experimental Psychology: Learning, Memory, and Cognition, 10, $723-732$.

Pinet, S., Ziegler, J. C., \& Alario, F. X. (2016). Typing is writing: Linguistic properties modulate typing execution. Psychonomic Bulletin \& Review, 23, 1898-1906.

Pylyshyn, Z. W. (1973). What the mind's eye tells the mind's brain: A critique of mental imagery. Psychological Bulletin, 80, 1-24.

Rosenbaum, D. A., Loukopoulos, L. D., Meulenbroek, R. G., Vaughan, J., \& Engelbrecht, S. E. (1995). Planning reaches by evaluating stored postures. Psychological Review, 102(1), 28-67.

Scaltritti, M., Arfe, B., Torrance, M., \& Peressotti, F. (2016). Typing pictures: Linguistic processing cascades into finger movements. Cognition, 156, 16-29.

Shaffer, L. H., \& Hardwick, J. (1968). Typing performance as a function of text. Quarterly Journal of Experimental Psychology, 20, 360369. doi:10.1080/14640746808400175

Shelton, A. L., \& McNamara, T. P. (2001). Systems of spatial reference in human memory. Cognitive Psychology, 43, 274-310.

Siegel, A. W., \& White, S. H. (1975). The development of spatial representations of large-scale environments. In H. W. Reese (Ed.),
Advances in child development and behavior (Vol. 10, pp. 9-55). Bingley: JAI.

Snyder, K. M., Ashitaka, Y., Shimada, H., Ulrich, J. E., \& Logan, G. D. (2014). What skilled typists don't know about the QWERTY keyboard. Attention, Perception, \& Psychophysics, 76, 162-171. doi: 10.3758/s13414-013-0548-4

Snyder, K. M., Logan, G. D., \& Yamaguchi, M. (2015). Watch what you type: The role of visual feedback from the screen and hands in skilled typewriting. Attention, Perception, \& Psychophysics, 77, 282-292. doi:10.3758/s13414-014-0756-6

Stevens, A., \& Coupe, P. (1978). Distortions in judged spatial relations. Cognitive Psychology, 10, 422-437.

Thorndyke, P. W., \& Hayes-Roth, B. (1982). Differences in spatial knowledge acquired from maps and navigation. Cognitive Psychology, 14, 560-589.

Tversky, B. (1993). Cognitive maps, cognitive collages, and spatial mental models. In A. U. Frank \& I. Campari (Eds.), Spatial information theory: A theoretical basis for GIS [COSIT 1993: Lecture notes in computer science] (Vol. 716, pp. 14-24). Berlin: Springer.

Tversky, B. (2000). Levels and structure of spatial knowledge. In R. Kitchin \& S. Freundschuh (Eds.), Cognitive mapping: Past, present and future (pp. 24 43). London: Routledge.

Van Selst, M. V., \& Jolicoeur, P. (1994). A solution to the effect of sample size on outlier elimination. The Quarterly Journal of Experimental Psychology, 47(3), 631-650.

Wang, R. F., \& Brockmole, J. R. (2003). Human navigation in nested environments. Journal of Experimental Psychology: Learning, Memory, and Cognition, 29, 398-404.

Wilson, M. (1987). UWA psychology: MRC psycholinguistic database (Dict interface). Retrieved from http://websites.psychology.uwa. edu.au/school/MRCDatabase/uwa_mrc.htm 